\title{
Effects of Tai Chi versus Proprioception Exercise Program on Neuromuscular Function of the Ankle in Elderly People: A Randomized Controlled Trial
}

\author{
Jing Liu, ${ }^{1}$ Xue-Qiang Wang, ${ }^{2}$ Jie-Jiao Zheng, ${ }^{3}$ Yu-Jian Pan, ${ }^{3}$ Ying-Hui Hua, ${ }^{4}$ \\ Shang-Min Zhao, ${ }^{3}$ Li-Yan Shen, ${ }^{3}$ Shuai Fan, ${ }^{2}$ and Jiu-Gen Zhong ${ }^{2}$ \\ ${ }^{1}$ College of Chinese Wushu, Shanghai University of Sport, Yangpu, Shanghai 200438, China \\ ${ }^{2}$ Department of Sport Rehabilitation, Shanghai University of Sport, Yangpu, Shanghai 200438, China \\ ${ }^{3}$ Department of Rehabilitation Medicine, Huadong Hospital, Fudan University, Shanghai 200040, China \\ ${ }^{4}$ Department of Sports Medicine, Huashan Hospital, Fudan University, Shanghai 200040, China
}

Correspondence should be addressed to Xue-Qiang Wang, qiang897@163.com and Jie-Jiao Zheng, zjjcss@126.com

Received 26 July 2012; Accepted 20 November 2012

Academic Editor: Annie Shirwaikar

Copyright (C) 2012 Jing Liu et al. This is an open access article distributed under the Creative Commons Attribution License, which permits unrestricted use, distribution, and reproduction in any medium, provided the original work is properly cited.

\begin{abstract}
Background. Tai Chi is a traditional Chinese medicine exercise used for improving neuromuscular function. This study aimed to investigate the effects of Tai Chi versus proprioception exercise program on neuromuscular function of the ankle in elderly people. Methods. Sixty elderly subjects were randomly allocated into three groups of 20 subjects per group. For 16 consecutive weeks, subjects participated in Tai Chi, proprioception exercise, or no structured exercise. Primary outcome measures included joint position sense and muscle strength of ankle. Subjects completed a satisfaction questionnaire upon study completion in Tai Chi and proprioception groups. Results. (1) Both Tai Chi group and proprioception exercise group were significantly better than control group in joint position sense of ankle, and there were no significant differences in joint position sense of ankle between TC group and PE group. (2) There were no significant differences in muscle strength of ankle among groups. (3) Subjects expressed more satisfaction with Tai Chi than with proprioception exercise program. Conclusions. None of the outcome measures on neuromuscular function at the ankle showed significant change posttraining in the two structured exercise groups. However, the subjects expressed more interest in and satisfaction with Tai Chi than proprioception exercise.
\end{abstract}

\section{Introduction}

An increasingly aging population presents a global challenge to human society. Such a population shift arises from two demographic effects: increasing longevity and declining fertility $[1,2]$. Based on World Health Organization projections, the proportion of the global population that is $60+$ years old is expected to increase from $10.0 \%$ in 2000 to $21.8 \%$ in 2050 , and then to rise to $32.2 \%$ in 2100 [3]. Due to decreased fertility, China and many other developing countries are going through more rapid fertility transitions than these projections predict. These countries will experience even faster population aging in future years than currently developed countries $[3,4]$.
Impaired motor performance in elderly people is often characterized by a slowing of movement, a decrease in muscle strength, and a loss of fine motor coordination [5]. These impairments can increase the likelihood of fall in elderly people, as well as decrease these individuals' ability to participate in standard activities of daily living $[6,7]$. Thus, realizing an effective method of improving the neuromuscular function of elderly people could be expected to improve quality of life and to reduce social medical costs [8].

Physical activity is an effective strategy for improving neuromuscular function, particularly among the elderly population [5, 8-10]. However, many forms of physical activity are either too intense or too monotonous for older adults to maintain over an extended period of time. 
Tai Chi (TC) is a popular form of exercise among older adults, especially in Asia. This activity involves a series of slow, smooth, and graceful movements, with an emphasis on smooth coordination of the eyes, head, body, and upper and lower extremities; for these reasons, TC is assigned special significance in the daily routine in many older adults [11]. Elderly TC learners may chat and learn from each other as they practice these skills, which can increase learning motivation and maintain a steady exercise habit. In addition, TC learners can practice by themselves at any time and in any place. An added benefit is that TC programs do not require any special equipment, which further adds to this activity's convenience and accessibility.

Numerous studies have investigated TC as an intervention for a wide variety of health problems, especially balance and musculoskeletal disease [12-14]. Li and his colleagues conduct a rigorous, multicenter randomized controlled trial with a large sample size in the New England Journal of Medicine, and they found that TC was more effective in improving postural stability in limits-of-stability tasks than a resistance-based exercise or low-impact stretching [15]. However, it is not yet known whether a proprioception exercise (PE) program has better long-term effects on the neuromuscular function of the ankle than a TC program. Ankle function is of special concern to older adults, as failure of this joint can lead to balance problems and increased risk of fall. Hence, we designed the present study to examine the effects of a 16-week TC training program versus 16 weeks of $\mathrm{PE}$ on the proprioceptive function and muscle strength of the ankle in elderly subjects. Here, we hypothesized that a TC program would lead to greater improvements in proprioceptive function and muscle strength outcomes than an alternative PE program.

\section{Methods}

2.1. Subjects. Subjects $(n=60)$ were recruited from several community elderly centers in Shanghai, China. Subjects were required to have had no previous experience in TC, as well as no regular physical exercise habits. A computer-generated random-number sequence randomly assigned all subjects to either a TC $(n=20$; mean age $\pm \mathrm{SD}, 70.5 \pm 2.1 \mathrm{y})$, PE $(n=20$; mean age $\pm \mathrm{SD}, 72.8 \pm 2.3 \mathrm{y})$, or control group $(n=20$; mean age $\pm \mathrm{SD}, 68.6 \pm 1.6 \mathrm{y})$. The TC group and the PE group both performed the assigned exercise twice a week ( $45 \mathrm{~min} / \mathrm{session}$ ) for 16 weeks. The control group had no regular physical exercise habits.

Inclusion criteria are the following: (1) be aged 60-85 years old; (2) score at least 24 on the Mini-Mental State Examination (MMSE) to show that they had no cognitive impairment [16], using the Chinese version validated by Chiu et al. (1994) [17]; (3) demonstrate a sufficient active range of motion in their upper limbs to perform the requisite finger-pointing tasks, which required subjects to flex and extend their shoulder, elbow, wrist, and fingers; (4) demonstrate through the Independent Activity of Daily Living test that they could be considered independent in activities of daily living [18].
Exclusion criteria are the following: (1) cardiovascular pathologies such as symptomatic cardiovascular disease or uncontrolled hypertension; (2) previous experience in TC; (3) any musculoskeletal disease referred to the lower limbs such as low back pain, serious arthritis, and ankylosing spondylitis; (4) any pathology affecting lower extremity function such as stroke, Parkinson's disease, or any other disabling neurologic illness.

2.2. Procedure. Prior to initiation of the study, all subjects completed a questionnaire that asked for such details as the subjects' past and present job status and their medical history. They also completed the MMSE and the Activity of Daily Living test and described their exercise habits (frequency and time/session). TC and PE subjects were asked to exercise twice a week ( $45 \mathrm{~min} / \mathrm{session}$ ) for 16 weeks at the Shanghai University of Sport. Joint position matching and muscle strength tests were conducted at baseline and after the 16-week intervention. Subjects completed a satisfaction survey after completing the 16-week intervention.

2.3. Testing Protocol. The assessment test was divided into two sections. The first section assessed subjects' joint position matching ability of the ankle in different degrees. Then, after 30 min of relaxation, the strength of the subjects' ankle dorsiflexors and plantar flexors was evaluated. All tests were performed on both legs of each subject.

Subjects performed a submaximal warm-up exercise $(50-60 \mathrm{~W})$ on a bicycle ergometer (MOTOmed viva2, Reck, Germany) for $5 \mathrm{~min}$ prior to the muscle tests. A Biodex System 3 isokinetic dynamometer (Biodex Medical Systems, Shirley, New York, USA) was used to measure peak torque, peak torque/weight, and ankle joint position sense (JPS).

2.3.1. Ankle Joint Position Passive Matching Test [19]. Each subject was positioned semirecumbent on the associated special testing chair, with the calf of the tested leg resting on a $40 \mathrm{~cm}$ high platform. The hip and knee were positioned at a $45^{\circ}$ flexion, and the talocrural joint was in neutral position. The bare foot of the subject was aligned with the axis of the dynamometer and attached to the footplate by a very small wrap to reduce cutaneous receptor input. During testing, subjects kept their eyes closed and wore headphones with music playing to eliminate visual and auditory stimuli from the testing apparatus. There were two reference degrees: (1) ankle at $10^{\circ}$ inversion and (2) ankle at $20^{\circ}$ inversion.

The subject's foot was first passively moved by the investigator to the maximal inversion or eversion position. The investigator then moved the foot to the two reference positions. This test position was maintained for $10 \mathrm{~s}$, with each subject instructed to concentrate on the position of the foot. The foot was then passively brought to maximal inversion or eversion and moved passively back toward eversion or inversion with a speed of $1 \%$ s. The subject was instructed to push on a stop button when he or she thought that the test position had been reached. This trial was repeated three times, and the error with which the subject reproduced the initial position was subsequently 
calculated. The three absolute error values were averaged, and the average value was termed the absolute angle error.

2.3.2. Muscle Strength Test [20]. We used a Biodex System 3 dynamometer to determine isokinetic peak torque and peak torque/weight values for reciprocal concentric plantar flexion to dorsiflexion movements of the ankle. Subjects were tested in a semi-recumbent position with $30^{\circ}$ of seatback tilt. The ankle was in $10^{\circ}$ plantar flexion. The knee of the tested ankle was in extension to minimize substitution from the hamstrings and other tibial rotators. Dynamometer and chair adjustments were made to align the midline of the foot with the midline of the patella. Two straps were wrapped around the extremity proximal to the patella and the pelvis to minimize movements of the hip and knee during testing. Isokinetic contractions were performed at an angular velocity of $30^{\circ} \%$ s.

Prior to testing, each subject performed a warm-up exercise of three submaximal repetitions to familiarize themselves with the equipment. For the isokinetic test, the subjects were instructed to push the foot away from them and pull it toward them at maximum velocity through the full available range of motion for each repetition. Peak torque was determined as the highest torque generated from the three trials. Peak torque/body weight is an important consideration for improved comparison among subjects of varied body types. None of the subjects noted any discomfort while testing.

\subsection{Training Program}

2.4.1. The TC Intervention. Subjects participated in $45 \mathrm{~min}$ TC sessions twice weekly for 16 weeks. Classes were taught by a Tai Chi master with more than 10 years of teaching experience. In the first session, we explained TC theory and procedures and provided subjects with printed teaching materials, including TC principles, practicing techniques, and safety precautions for the elderly. For the remaining sessions, each subject practiced TC under the instruction of the Tai Chi master. Each session included (1) $5 \mathrm{~min}$ of warm-up and a review of TC principles, (2) $30 \mathrm{~min}$ of TC movement, (3) 5 min of breathing techniques, and (4) $5 \mathrm{~min}$ of cooldown. The program consisted of 24 forms from classic Yang-style Tai Chi, with minor modifications that were suitable for older adults. We encouraged subjects to participate in their usual sports activities, but to not engage in extra strength training.

2.4.2. The Proprioceptive Exercise Intervention. Proprioceptive exercise classes were led by a registered physical therapist for $45 \mathrm{~min}$, twice per week, over 16 weeks. The exercise protocol emphasized static and dynamic balance exercises, including transitions between differing sensory conditions and functional everyday movements. Each lesson incorporated a similar general plan as follows: (1) 5 min warm-up; (2) 20 min of static balance exercises such as squats (two-leg stance) and one-leg stance; (3) $15 \mathrm{~min}$ of dynamic balance exercises such as jogging, sideways walking or running with crossovers, forward walking or running in a zigzag line, or backward walking or running in a zigzag line; (4) 5 min of cool-down. Exercises gradually increased in difficulty and training load over the 16-week training period.

2.5. Statistical Analyses. Statistical analyses were performed using SPSS 17.0 and Microsoft Excel 2003 software. Data are expressed as mean $\pm \mathrm{SD}$. Changes in variables between preand posttraining and between groups were analyzed. A oneway analysis of variance (ANOVA) was used to examine the differences among the characteristics at baseline of the TC, $\mathrm{PE}$, and control groups. Changes in the JPS, peak torque, and peak torque/weight between baseline and followup among the TC, PE, and control groups were compared using twoway (3 groups $\times 2$ repetition assessments) repeated measures ANOVA. When ANOVA analysis revealed significant time and time-by-group interaction effects, paired $t$-tests were used to compare the changes in measures within groups. Statistical significance was assumed at $P$ less than 0.05 .

We used five-point Likert scales to contrast the differences between the TC and PE groups with regard to health satisfaction and recommending others to participate in the project after 16 weeks of intervention [21]. With five-point scales, the points could be labeled as (1) 1: very satisfied; 2 : satisfied; 3: neither satisfied nor dissatisfied; 4: dissatisfied; 5: very dissatisfied for health satisfaction; and (2) 1: strongly agree; 2: somewhat agree; 3 : neutral/no opinion; 4: somewhat disagree; 5: strongly disagree about recommending that other people participate in the program. The Mann-Whitney Test was used to compare the differences between groups. Statistical significance was assumed at $P$ less than 0.05 .

\section{Results}

The study design is outlined in Figure 1. Twelve of the 72 individuals initially recruited were deemed ineligible for study participation; eight subjects did not meet the inclusion criteria, two subjects had previous Tai Chi experience, one subject's work schedule was incompatible, and one subject withdrew consent. A total of 42 subjects completed the 16week study program, and 18 subjects (TC $n=5, \operatorname{PE~} n=10$, control $n=3$ ) were lost to followup. Reasons for dropout included illness, withdrawal, serious family problems, and not attending the final evaluation session. Hence, followup data were available for 15 of the 20 subjects in the TC group, 10 of the 20 subjects in the PE group, and 17 of the 20 subjects in the control group. Table 1 lists baseline characteristics of the three groups. The groups were well matched at the baseline assessment, with no differences in key outcome variables apparent.

3.1. Ankle Joint Position Sense. For the absolute error after the 16-week program (see Figure 2), (1) subjects in the TC group and $\mathrm{PE}$ group could be matching significantly more accurate than those in the control group $(P=0.014$ for TC, $P=0.039$ for PE) in the left ankle, (2) subjects in the TC group and PE group had a smaller amount of absolute error in the right ankle than the control group, but there were no significant differences $(P=0.184$ for TC, $P=0.883$ for PE), 


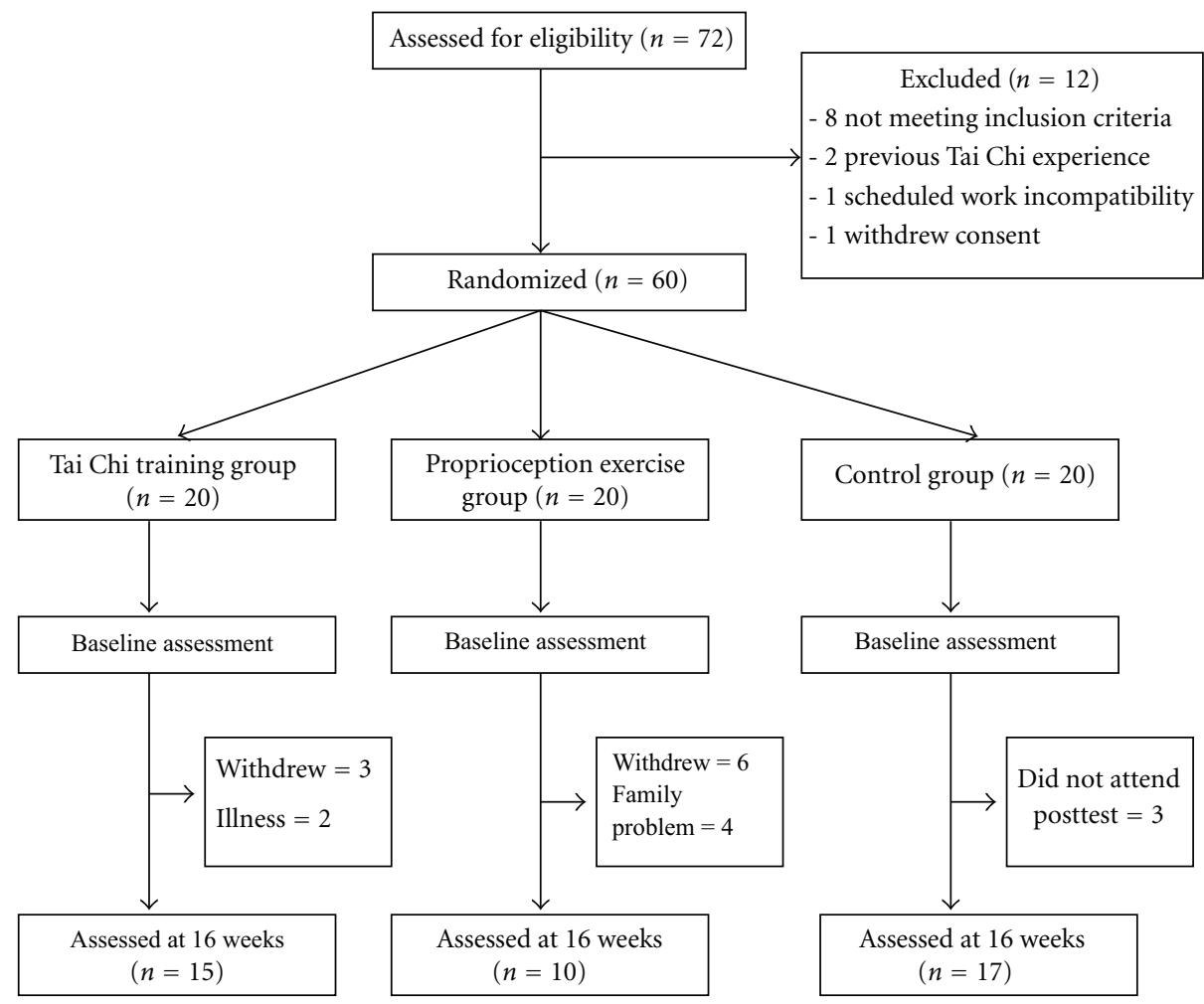

FIGURE 1: Flow diagram of eligibility assessment, exclusion, inclusion, and analysis.

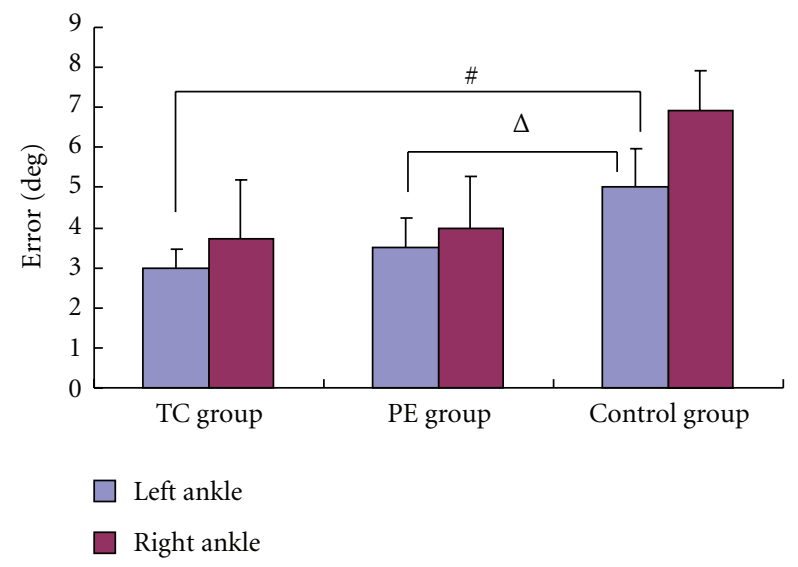

Figure 2: Joint position sense for 3 groups. \#There were significant differences between TC group and control group, $P=0.011$. ${ }^{\Delta}$ There were significant differences between PE group and control group, $P=0.045$. TC: Tai Chi; PE: proprioception exercise.

and (3) there were no significant differences in joint position matching of both ankles for the TC group and PE group $(P=$ 0.979 for left, $P=0.184$ for right).

3.2. Muscle Strength. We found no significant differences in the strength and endurance of the bilateral ankle dorsiflexors and plantar flexors at a speed of $30 \%$ s for concentric test conditions among groups (Table 2).
3.3. Satisfaction Survey. Elderly subjects expressed significantly higher health satisfaction in the TC group than in the PE group after 16 weeks $(P=0.036)$, and more subjects in the TC group recommended the program than did those in the PE group (Figures 3 and 4 ).

\section{Discussion}

4.1. Joint Position Sense. The TC and PE interventions evoked improvements in the joint position sense of bilateral ankles in this study. However, we were unable to show a difference after a 16-week exercise intervention between the TC group and PE group on a joint position sense. Leung et al. [22] performed a systematic and meta-analytical review of the effects of TC on balance function in elderly people. The systematic review based on 13 randomized controlled trials (RCTs) indicated that TC was effective in improving the balance function of elderly people. However, a TC program may not necessarily be superior to other interventions. Lelard et al. [10] assessed the effects of a TC program versus a balance training program on postural control and walking ability in elderly people. The authors measured static postural control and walking speed. They also did not find any significant modifications of postural parameters and walking speed between TC and balance training. Indeed, studies under more challenging postural conditions should be performed to verify this specific effect of TC training on proprioception function of the ankle in the daily living activities of older adults. 


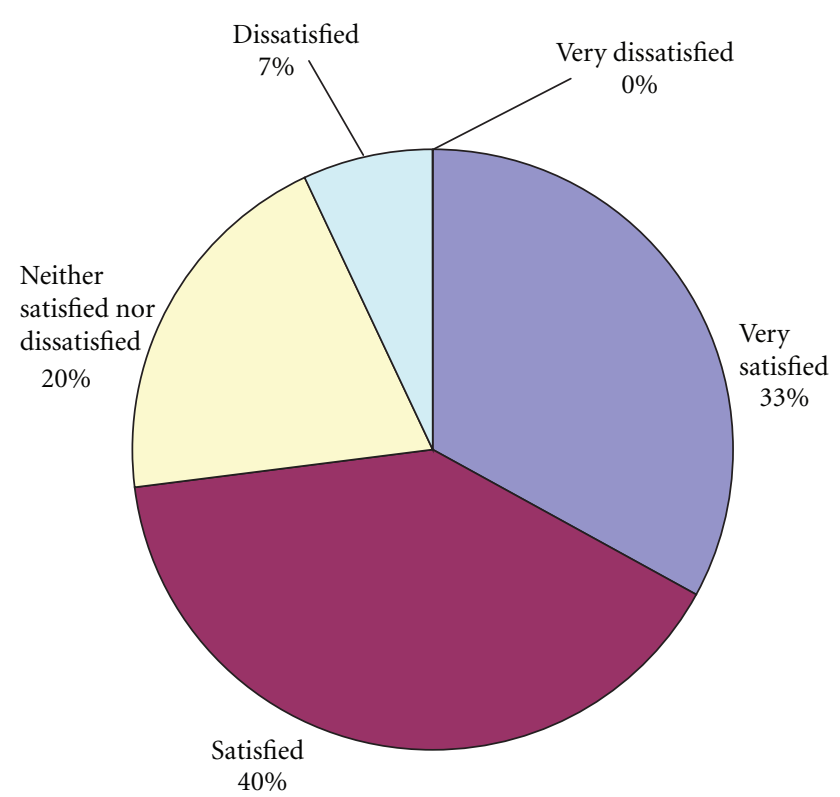

(a)

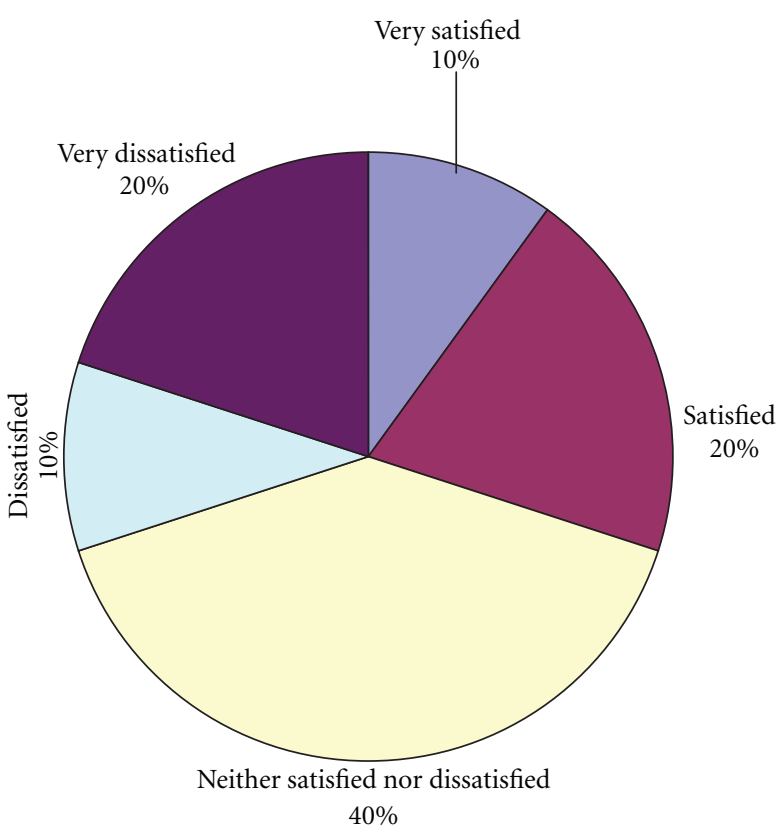

(b)

Figure 3: (a) Health satisfaction for Tai Chi group. (b) Health satisfaction for proprioception exercise group. The Mann-Whitney Test was used to compare the differences between groups. There were significant differences between PE group and control group, $P=0.036$.

TABLE 1: Baseline values for the 3 groups.

\begin{tabular}{|c|c|c|c|c|}
\hline Variable & $\mathrm{TC}(n=15)$ & $\mathrm{PE}(n=10)$ & Control group $(n=17)$ & $P$ \\
\hline Men/female & $7 / 8$ & $4 / 6$ & $7 / 10$ & $0.932 *$ \\
\hline Age (y) & $68.0 \pm 1.41$ & $68.8 \pm 1.03$ & $69.8 \pm 0.73$ & $0.663^{\#}$ \\
\hline Body weight (kg) & $60.73 \pm 2.14$ & $55.60 \pm 2.73$ & $59.59 \pm 1.45$ & $0.252^{\#}$ \\
\hline Height $(\mathrm{cm})$ & $164.33 \pm 2.09$ & $167.30 \pm 1.51$ & $165.28 \pm 0.95$ & $0.528^{\#}$ \\
\hline \multicolumn{5}{|l|}{ JPS test $\left({ }^{\circ}\right)$} \\
\hline Left ankle & $4.20 \pm 0.75$ & $6.13 \pm 1.77$ & $4.16 \pm 0.72$ & $0.431^{\#}$ \\
\hline Right ankle & $8.93 \pm 1.62$ & $7.40 \pm 1.43$ & $8.77 \pm 1.28$ & $0.785^{\#}$ \\
\hline \multicolumn{5}{|l|}{$\mathrm{PT}(\mathrm{Nm})$ : concentric, $30^{\circ} / \mathrm{s}$} \\
\hline Left plantar flexion & $49.88 \pm 6.81$ & $46.68 \pm 6.97$ & $49.62 \pm 4.59$ & $0.934^{\#}$ \\
\hline Right plantar flexion & $49.08 \pm 7.13$ & $54.64 \pm 8.03$ & $55.55 \pm 4.86$ & $0.729^{\#}$ \\
\hline Left dorsiflexion & $14.4 \pm 1.32$ & $14.98 \pm 2.36$ & $14.60 \pm 1.41$ & $0.975^{\#}$ \\
\hline Right dorsiflexion & $14.75 \pm 1.45$ & $17.10 \pm 2.63$ & $16.95 \pm 1.72$ & $0.630^{\#}$ \\
\hline \multicolumn{5}{|c|}{ PT/weight $(\mathrm{Nm} / \mathrm{kg})$ : concentric, $30^{\circ} / \mathrm{s}$} \\
\hline Left plantar flexion & $0.82 \pm 0.09$ & $0.85 \pm 0.13$ & $0.84 \pm 0.082$ & $0.978^{\#}$ \\
\hline Right plantar flexion & $0.82 \pm 0.11$ & $0.99 \pm 0.14$ & $0.95 \pm 0.91$ & $0.534^{\#}$ \\
\hline Left dorsiflexion & $0.24 \pm 0.02$ & $0.27 \pm 0.04$ & $0.24 \pm 0.02$ & $0.782^{\#}$ \\
\hline Right dorsiflexion & $0.25 \pm 0.02$ & $0.31 \pm 0.04$ & $0.29 \pm 0.03$ & $0.467^{\#}$ \\
\hline
\end{tabular}

Data reported as mean $\pm \mathrm{SD}$.

*Chi-square test.

\#One-way analysis of variance.

TC: tai chi; PE: proprioception exercise; JPS: joint position sense; PT: peak torque.

In addition, we found that the joint position sense of the left ankle was better than the right ankle in baseline values for the three groups. Significant matching of subjects in the TC group and PE group could be achieved more accurately than those in the control group $(P=0.014$ for TC, $P=0.039$ for PE) in the left ankle after 16 weeks of exercise. The result was similar to those reported previously in several studies [23-25]. Goble and Brown [23] found that for the proprioceptive matching task, errors were smaller for the nonpreferred left arm, whereas during the visual matching task, smaller errors were found for the preferred right arm. These results suggest a left-arm/right-hemisphere 


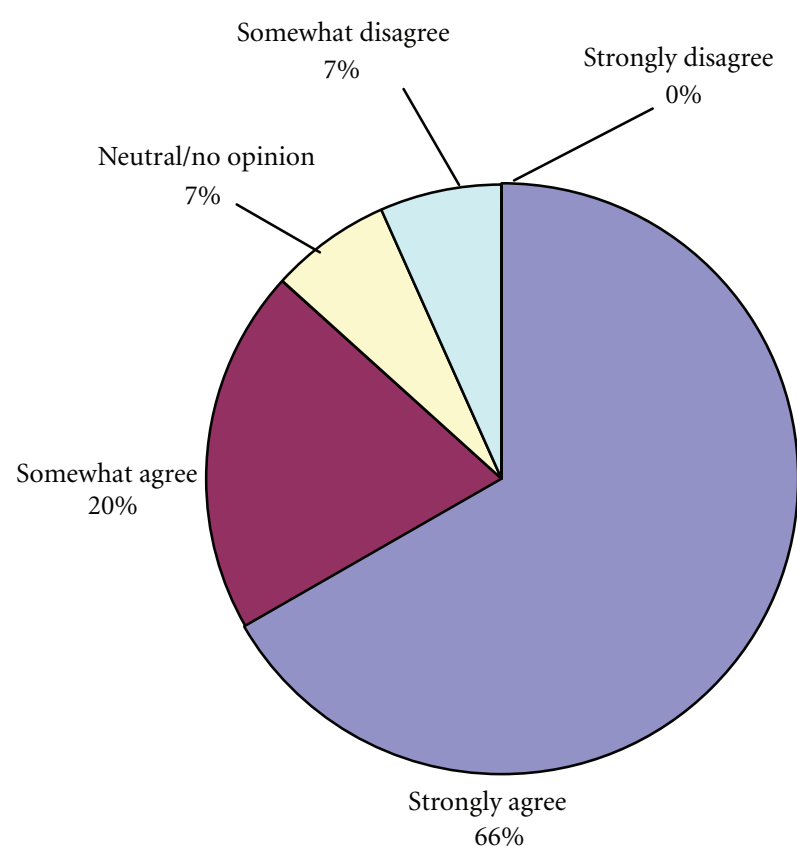

(a)

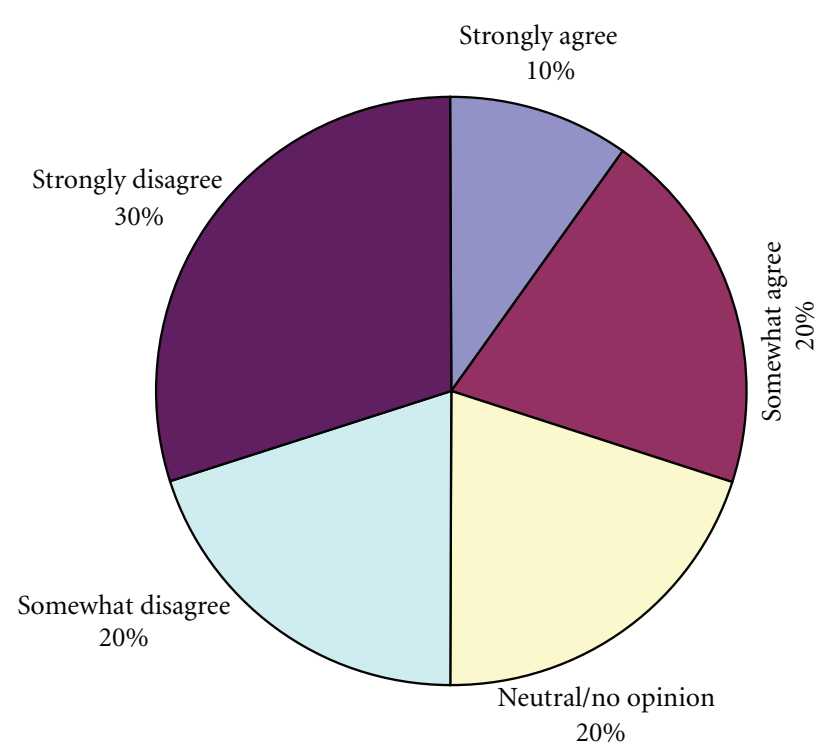

(b)

FIgURE 4: (a) Recommending other people learn the program for tai chi group. (b) Recommending other people learn the program for proprioception exercise group. The Mann-Whitney Test was used to compare the differences between groups. There were significant differences between PE group and control group, $P=0.002$.

TABLE 2: Muscle strength for the 3 groups.

\begin{tabular}{|c|c|c|c|c|}
\hline Variable & TC & $\mathrm{PE}$ & Control group & $P^{*}$ \\
\hline \multicolumn{5}{|l|}{ JPS test $\left({ }^{\circ}\right)$} \\
\hline Left ankle & $3.0 \pm 0.48^{\#}$ & $3.5 \pm 0.72 \triangle$ & $5.03 \pm 0.95$ & 0.026 \\
\hline Right ankle & $3.7 \pm 1.49$ & $4.0 \pm 1.29$ & $6.91 \pm 0.99$ & 0.083 \\
\hline \multicolumn{5}{|c|}{$\mathrm{PT}(\mathrm{Nm})$ : concentric, $30 /{ }^{\circ} \mathrm{s}$} \\
\hline Left plantar flexion & $59.16 \pm 6.71$ & $61.4 \pm 6.09$ & $47.76 \pm 3.69$ & 0.138 \\
\hline Right plantar flexion & $61.52 \pm 7.72$ & $69.53 \pm 9.25$ & $52.73 \pm 4.37$ & 0.221 \\
\hline Left dorsiflexion & $17.84 \pm 2.04$ & $16.93 \pm 2.71$ & $13.11 \pm 6.15$ & 0.128 \\
\hline Right dorsiflexion & $14.87 \pm 1.37$ & $18.02 \pm 3.31$ & $14.88 \pm 1.37$ & 0.079 \\
\hline \multicolumn{5}{|c|}{$\mathrm{PT} /$ weight $(\mathrm{Nm} / \mathrm{kg})$ : concentric, $30 /{ }^{\circ} \mathrm{s}$} \\
\hline Left plantar flexion & $0.98 \pm 0.1$ & $1.13 \pm 0.1$ & $0.82 \pm 0.04$ & 0.11 \\
\hline Right plantar flexion & $1.01 \pm 0.1$ & $1.25 \pm 0.1$ & $0.92 \pm 0.1$ & 0.19 \\
\hline Left dorsiflexion & $0.31 \pm 0.04$ & $0.31 \pm 0.05$ & $0.22 \pm 0.03$ & 0.197 \\
\hline Right dorsiflexion & $0.34 \pm 0.03$ & $0.32 \pm 0.05$ & $0.26 \pm 0.03$ & 0.150 \\
\hline
\end{tabular}

Data reported as mean $\pm \mathrm{SD}$.

${ }^{*}$ A 2-way analysis of variance (group $\times$ time). ${ }^{*}$ There was significant differences between TC group and control group, $P=0.011$. $\triangle$ There was significant differences between PE group and control group, $P=0.045$.

TC: tai chi; PE: proprioception exercise; PT: peak torque.

advantage for proprioceptive feedback processing and a right-arm/left-hemisphere advantage for visual information processing. Such asymmetries may reflect fundamental differences between the two-arm/hemisphere systems during the performance of bimanual tasks in which the preferred arm requires visual guidance to manipulate an object, whereas the nonpreferred limb stabilizes that object on the basis of proprioceptive feedback. The result of our study also showed that joint position sense of the left ankle was more sensitive to TC training and to the PE program than the right ankle.

4.2. Muscle Strength. Many researchers reported only mean peak torque values rather than values normalized by body weight. Normalizing by body weight is, thus, an important consideration for better comparison among subjects of 
varied body types. Additionally, as ankle sprains most usually occur in the closed kinetic chain, body weight also has an influence on the sprain moment generated at the ankle. Therefore, we consider peak torque/body weight a more relevant value compared with peak torque.

Our results affirm that subjects in the TC and PE groups did not have a larger amount of peak torque and peak torque/weight in both ankles than the control group, and there were no significant differences between groups. Perhaps, in order to improve muscle strength of the ankle joint, a 16-week TC intervention might not last long enough. For the lower limb muscle test, our results were consistent with previous studies [26-28], which showed that muscle strength of a TC program group for training lasting less than a year was not significantly higher than that of the control group. Wolf et al. [29] found that TC and elderly balance training participants with four months of training had no differences in ankle muscle strength. Another study confirmed that long-term TC exercisers with more than four years of experience showed significantly better muscle strength of the ankle joint compared with long-term regular joggers/swimmers and sedentary elderly people [30]. The results of these papers suggest that improving biomechanical characteristics of lower-extremity muscles may require a longer-lasting TC intervention for elderly people.

4.3. Satisfaction Survey. We did not find that the PE program has an improved effect than the TC program on proprioceptive function or muscle strength. However, the TC and PE groups showed a significant difference in health satisfaction and recommendation of the treatment to others after 16 weeks of practice. There may be several reasons for this result. First, a TC program is distinctive because it includes a structured cognitive component, also referred to as meditation [31]. In Tai Chi, a practitioner is required to choreograph slow movements according to visual imagery. In short, the mind directs the body in performing these movements. This makes TC different from other types of balance exercise.

Brown et al. [32] provided the hypothesis that exercise plus cognitive strategy training programs are more effective than exercise programs lacking a structured cognitive component in promoting psychological benefits. But in proprioception exercise without a structured cognitive component, the practitioner's cognitive processing is random and unstructured. And Silsupadol et al. [33] suggested that cognitive dual-task training programs (balance exercise + cognitive training) were superior to single-task training (balance exercise) in improving balance function. Second, a PE program is not usually sufficiently interesting that the majority of elderly participants will maintain a longterm and regular habit of exercise. Lastly, subjects felt comfortable with the intensity of the program, and none reported discomfort while practicing Tai Chi. Participants were enthusiastic and made every effort to attend sessions. Our TC program proved to be effective, interesting, and convenient as a form of physical activity for older adults.
4.4. Limitations. Although our study elicited important observations regarding the usefulness of $\mathrm{TC}$ on the ankle in elderly people, there are some limitations to our research. First, because subjects had not learned the movements of tai chi previously, it sometimes proved difficult for subjects to correctly perform this exercise. We note that the present findings cannot be generalized to elderly people living in nursing homes or hospital settings, as these individuals are more likely to have limited mobility and/or a preestablished exercise program that does not permit physical interventions such as those assessed here. Finally, the sample size was too small to draw any firm conclusions. Further rigorous, multicenter RCTs with a large sample size are warranted.

\section{Conclusion}

Results of our study demonstrated similar effects of 16-week TC and proprioception exercise programs on joint position sense or muscle strength of ankle joint in elderly people. None of the outcome measures showed significant change posttraining in the TC or proprioception exercise groups. However, the elderly felt significantly more interested in TC program than PE program and also significantly more satisfactory to their health in the TC group than the PE group. Further study with long-term followup is needed to substantiate the role of Tai Chi exercise in the physical and psychological benefits.

\section{Ethical Approval}

This project was approved by the ethics committee of the Shanghai University of Sport.

\section{Conflict of Interests}

The authors report no conflict of interests.

\section{Acknowledgments}

The authors thank the elderly adult centers for permission to recruit their subjects. This project was supported by the Natural Science Foundation of Shanghai, China (Grant no. 07ZR14103) and the National Natural Science Foundation of China (Grant no. 81101391). The trial was registered in Chinese Clinical Trial Registry (http://www.chictr.org/) (Registration no. ChiCTR-TRC-12002244).

\section{References}

[1] T. B. L. Kirkwood, "Global aging and the brain," Nutrition Reviews, vol. 68, supplement 2, pp. S65-S69, 2010.

[2] D. Harman, "Aging: overview," Annals of the New York Academy of Sciences, vol. 928, pp. 1-21, 2001.

[3] W. Lutz, W. Sanderson, and S. Scherbov, "The coming acceleration of global population ageing," Nature, vol. 451, no. 7179, pp. 716-719, 2008.

[4] Z. Yuan and X. Z. He, "Aging population and health inequalities in the rural areas of China," Journal of the American Geriatrics Society, vol. 58, no. 6, pp. 1210-1211, 2010. 
[5] S. M. Szabo, P. A. Janssen, K. Khan, M. J. Potter, and S. R. Lord, "Older women with age-related macular degeneration have a greater risk of falls: a physiological profile assessment study," Journal of the American Geriatrics Society, vol. 56, no. 5, pp. 800-807, 2008.

[6] R. J. Petrella, P. J. Lattanzio, and M. G. Nelson, "Effect of age and activity on knee joint proprioception," American Journal of Physical Medicine and Rehabilitation, vol. 76, no. 3, pp. 235241, 1997.

[7] N. C. Voermans, A. H. Snijders, Y. Schoon, and B. R. Bloem, "Why old people fall (and how to stop them)," Practical Neurology, vol. 7, no. 3, pp. 158-171, 2007.

[8] A. C. King and J. M. Guralnik, "Maximizing the potential of an aging population," Journal of the American Medical Association, vol. 304, no. 17, pp. 1944-1945, 2010.

[9] Y. K. Chang, Y. H. Nien, C. L. Tsai, and J. L. Etnier, "Physical activity and cognition in older adults: the potential of Tai Chi Chuan," Journal of Aging and Physical Activity, vol. 18, no. 4, pp. 451-472, 2010.

[10] T. Lelard, P. L. Doutrellot, P. David, and S. Ahmaidi, "Effects of a 12-week Tai Chi Chuan program versus a balance training program on postural control and walking ability in older people," Archives of Physical Medicine and Rehabilitation, vol. 91, no. 1, pp. 9-14, 2010.

[11] J. C. Kwok, C. W. Hui-Chan, and W. W. Tsang, "Effects of aging and Tai Chi on finger-pointing toward stationary and moving visual targets," Archives of Physical Medicine and Rehabilitation, vol. 91, no. 1, pp. 149-155, 2010.

[12] A. M. Kuramoto, "Therapeutic benefits of Tai Chi exercise: research review," Wisconsin Medical Journal, vol. 105, no. 7, pp. 42-46, 2006.

[13] R. E. Taylor-Piliae, W. L. Haskell, N. A. Stotts, and E. S. Froelicher, "Improvement in balance, strength, and flexibility after 12 weeks of Tai chi exercise in ethnic Chinese adults with cardiovascular disease risk factors," Alternative Therapies in Health and Medicine, vol. 12, no. 2, pp. 50-58, 2006.

[14] C. Lan, J. S. Lai, S. Y. Chen, and M. K. Wong, "12-month Tai Chi training in the elderly: its effect on health fitness," Medicine and Science in Sports and Exercise, vol. 30, no. 3, pp. 345-351, 1998.

[15] F. Li, P. Harmer, K. Fitzgerald et al., "Tai chi and postural stability in patients with Parkinson's disease," The New England Journal of Medicine, vol. 366, no. 6, pp. 511-519, 2012.

[16] M. F. Folstein, S. E. Folstein, and P. R. McHugh, "Mini mental state. A practical method for grading the cognitive state of patients for the clinician," Journal of Psychiatric Research, vol. 12, no. 3, pp. 189-198, 1975.

[17] H. F. Chiu, H. C. Lee, W. S. Chung et al., "Reliability and validity of the Cantonese version of mini-mental status examination: a preliminary study," Hong Kong Journal of Psychiatry, vol. 4, supplement 2, pp. 25-28, 1994.

[18] S. Katz, T. D. Downs, H. R. Cash, and R. C. Grotz, "Progress in development of the index of ADL," Gerontologist, vol. 10, no. 1, pp. 20-30, 1970.

[19] U. Sekir, Y. Yildiz, B. Hazneci, F. Ors, T. Saka, and T. Aydin, "Reliability of a functional test battery evaluating functionality, proprioception, and strength in recreational athletes with functional ankle instability," European Journal of Physical and Rehabilitation Medicine, vol. 44, no. 4, pp. 407415, 2008

[20] S. T. Aydog, L. Ozçakar, O. Tetik, H. A. Demirel, Z. Hasçelik, and M. N. Doral, "Relation between foot arch index and ankle strength in elite gymnasts: a preliminary study," British Journal of Sports Medicine, vol. 39, no. 3, article e13, 2005.
[21] R. Likert, "A technique for the measurement of attitudes," Archives of Psychology, vol. 22, pp. 1-55, 1932.

[22] D. P. Leung, C. K. Chan, H. W. Tsang et al., "Tai chi as an intervention to improve balance and reduce falls in older adults: a systematic and meta-analytical review," Alternative Therapies in Health and Medicine, vol. 17, no. 1, pp. 40-48, 2011.

[23] D. J. Goble and S. H. Brown, "Upper limb asymmetries in the matching of proprioceptive versus visual targets," Journal of Neurophysiology, vol. 99, no. 6, pp. 3063-3074, 2008.

[24] D. J. Goble and S. H. Brown, "Dynamic proprioceptive target matching behavior in the upper limb: effects of speed, task difficulty and arm/hemisphere asymmetries," Behavioural Brain Research, vol. 200, no. 1, pp. 7-14, 2009.

[25] D. J. Goble, B. C. Noble, and S. H. Brown, "Proprioceptive target matching asymmetries in left-handed individuals," Experimental Brain Research, vol. 197, no. 4, pp. 403-408, 2009.

[26] J. Woo, A. Hong, E. Lau, and H. Lynn, "A randomised controlled trial of Tai Chi and resistance exercise on bone health, muscle strength and balance in community-living elderly people," Age and Ageing, vol. 36, no. 3, pp. 262-268, 2007.

[27] R. Song, B. L. Roberts, E. O. Lee, P. Lam, and S. C. Bae, "A randomized study of the effects of Tai Chi on muscle strength, bone mineral density, and fear of falling in women with osteoarthritis," Journal of Alternative and Complementary Medicine, vol. 16, no. 3, pp. 227-233, 2010.

[28] J. X. Li, D. Q. Xu, and Y. Hong, "Changes in muscle strength, endurance, and reaction of the lower extremities with Tai Chi intervention," Journal of Biomechanics, vol. 42, no. 8, pp. 967971, 2009.

[29] S. L. Wolf, H. X. Barnhart, N. G. Kutner, E. McNeely, C. Coogler, and $\mathrm{T}$. $\mathrm{Xu}$, "Reducing frailty and falls in older persons: an investigation of Tai Chi and computerized balance training," Journal of the American Geriatrics Society, vol. 44, no. 5, pp. 489-497, 1996.

[30] D. Q. Xu, J. X. Li, and Y. Hong, "Effects of long term Tai Chi practice and jogging exercise on muscle strength and endurance in older people," British Journal of Sports Medicine, vol. 40, no. 1, pp. 50-54, 2006.

[31] L. Y. K. Lee, D. T. F. Lee, and J. Woo, "Tai Chi and healthrelated quality of life in nursing home residents," Journal of Nursing Scholarship, vol. 41, no. 1, pp. 35-43, 2009.

[32] D. R. Brown, Y. Wang, A. Ward et al., "Chronic psychological effects of exercise and exercise plus cognitive strategies," Medicine and Science in Sports and Exercise, vol. 27, no. 5, pp. 765-775, 1995.

[33] P. Silsupadol, A. Shumway-Cook, V. Lugade et al., "Effects of single-task versus dual-task training on balance performance in older adults: a double-blind, randomized controlled trial," Archives of Physical Medicine and Rehabilitation, vol. 90, no. 3, pp. 381-387, 2009. 


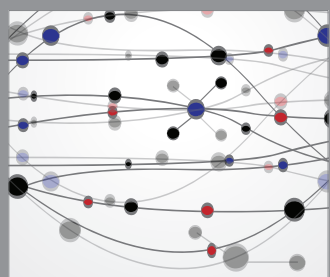

The Scientific World Journal
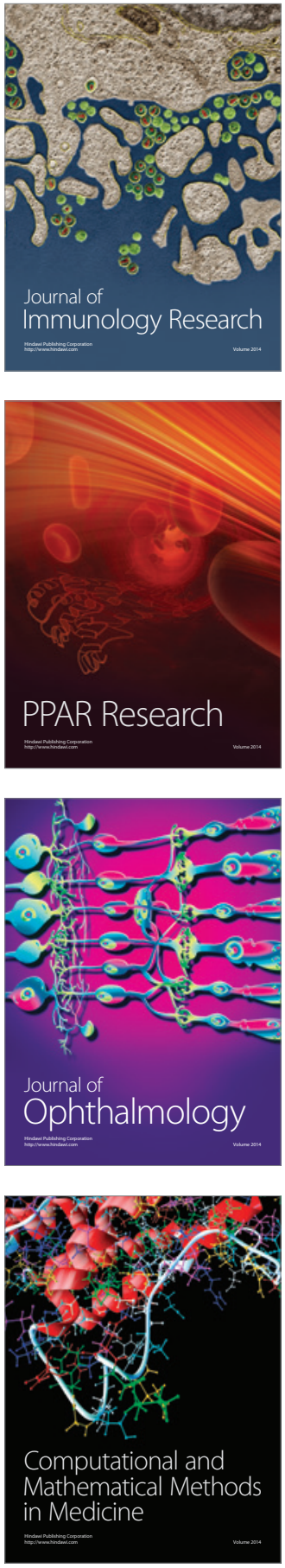

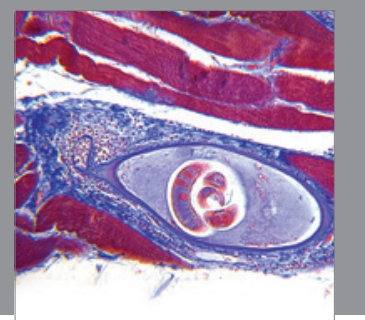

Gastroenterology

Research and Practice
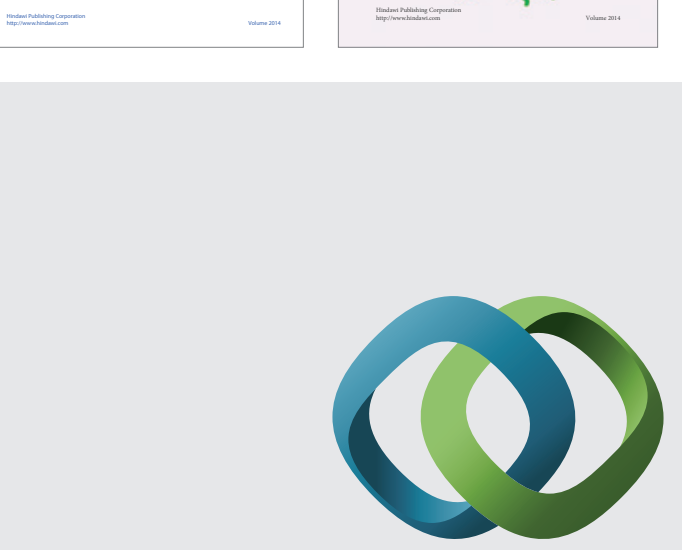

\section{Hindawi}

Submit your manuscripts at

http://www.hindawi.com
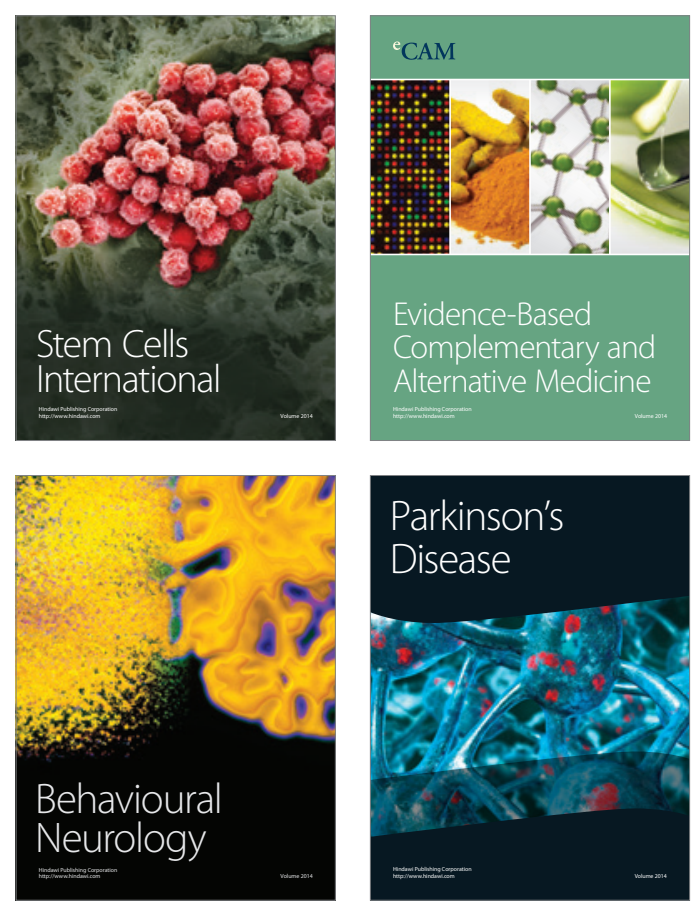

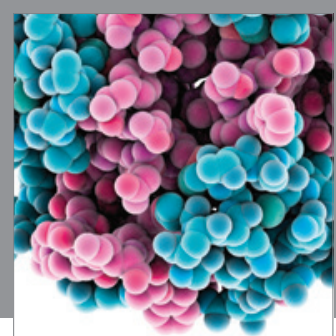

Journal of
Diabetes Research

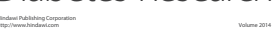

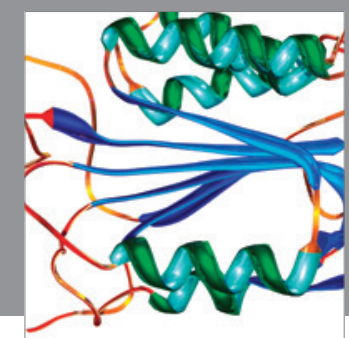

Disease Markers
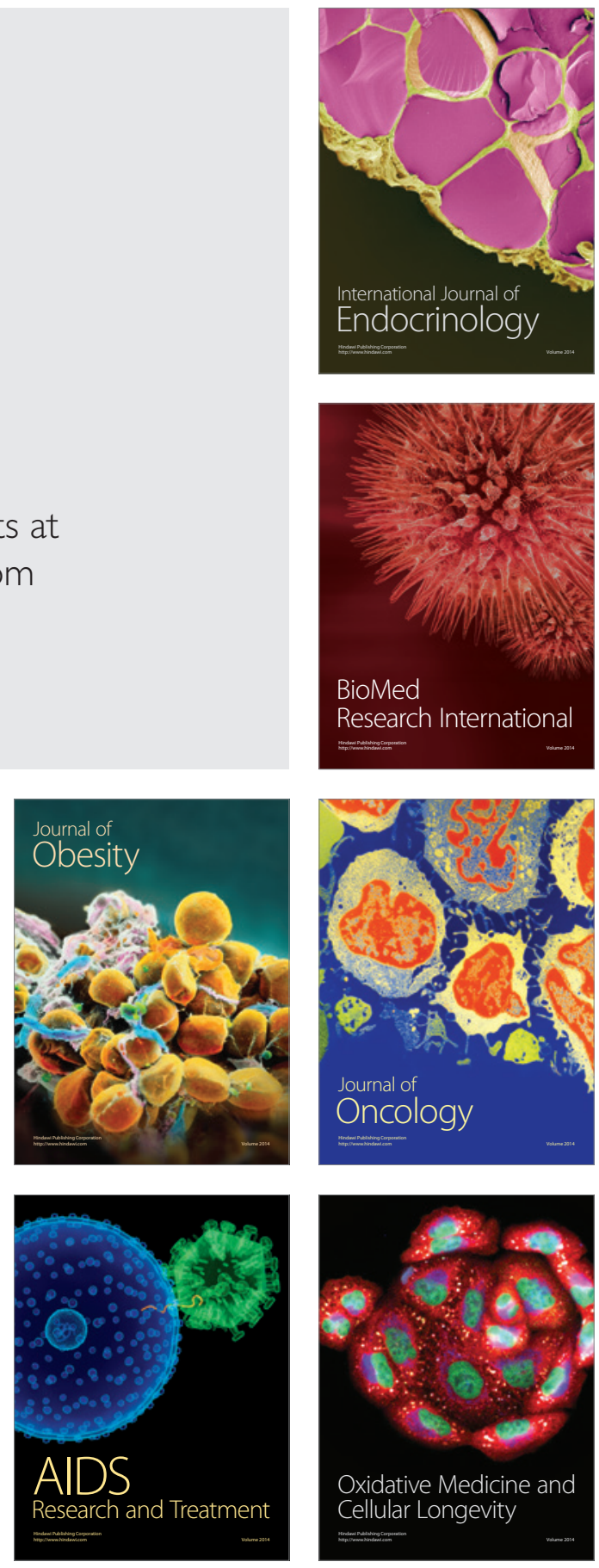